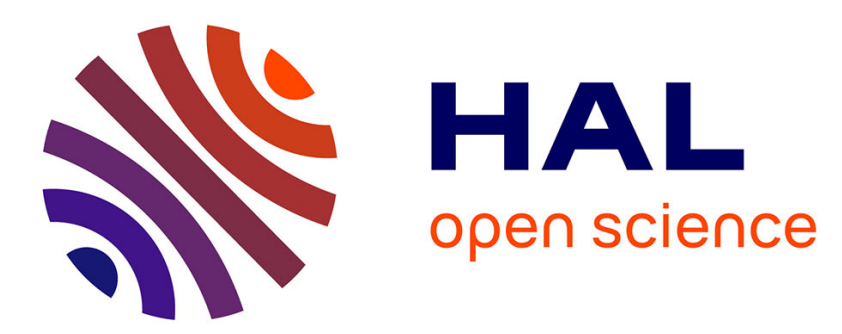

\title{
Adaptive Multitemporal SAR Image Filtering Based on the Change Detection Matrix
}

Thu Trang Le, Abdourrahmane Atto, Emmanuel Trouvé, Jean-Marie Nicolas

\section{To cite this version:}

Thu Trang Le, Abdourrahmane Atto, Emmanuel Trouvé, Jean-Marie Nicolas. Adaptive Multitemporal SAR Image Filtering Based on the Change Detection Matrix. IEEE Geoscience and Remote Sensing Letters, 2014, 11 (10), 5 p. 10.1109/LGRS.2014.2311663 . hal-00975385v2

\section{HAL Id: hal-00975385 \\ https://hal.science/hal-00975385v2}

Submitted on 11 Nov 2014

HAL is a multi-disciplinary open access archive for the deposit and dissemination of scientific research documents, whether they are published or not. The documents may come from teaching and research institutions in France or abroad, or from public or private research centers.
L'archive ouverte pluridisciplinaire HAL, est destinée au dépôt et à la diffusion de documents scientifiques de niveau recherche, publiés ou non, émanant des établissements d'enseignement et de recherche français ou étrangers, des laboratoires publics ou privés. 


\title{
Adaptive Multitemporal SAR Image Filtering Based on the Change Detection Matrix
}

\author{
Thu Trang Lê, Abdourrahmane M. Atto, Member, IEEE, Emmanuel Trouvé, Senior Member, IEEE, \\ and Jean-Marie Nicolas
}

\begin{abstract}
This letter presents an adaptive filtering approach of Synthetic Aperture Radar (SAR) image times series based on the analysis of the temporal evolution. First, change detection matrices (CDMs) containing information on changed and unchanged pixels are constructed for each spatial position over the time series by implementing coefficient of variation (CV) cross tests. Afterwards, the CDM provides for each pixel in each image, an adaptive spatiotemporal neighborhood which is used to derive the filtered value. The proposed approach is illustrated on a time series of 25 ascending TerraSAR-X images acquired from November 6, 2009 to September 25, 2011 over the ChamonixMontBlanc test-site, which includes different kinds of change, such as parking occupation, glacier surface evolution, etc.
\end{abstract}

Index Terms-Change detection, coefficient of variation (CV), SAR image time series, temporal adaptive filtering.

\section{INTRODUCTION}

The potentiality and utility of synthetic aperture radar (SAR) data are widely recognized for geoscience and remote sensing applications. In particular, the launch of new generation satellites makes it possible to obtain time series of SAR images with short repeat cycle and high resolution. One of the most important problems of SAR data is speckle noise involved in coherent acquisition systems, which makes it difficult for both human and automatic interpretation.

A large number of speckle filtering approaches has been proposed for single date SAR images (using spatial information only). An important issue of a filter is the sample selection for the filtering process. In this respect, we have the following:

1) Local approaches, which use the window/neighborhood of the considered pixel to estimate the filtered value. Different spatial estimation supports have been developed: 1) Boxcar window [1], [2], etc., in which optimal parameters are estimated by a fixed-size rectangular window sliding through each pixel of the image. This is the simplest analysis window, its disadvantage is that speckle reduction also impacts the edge sharpness. 2) Directional neighborhood: For the edge preservation purpose, estimation neighborhood near the high contrast area takes the orientation of the edge into account by incorporating the local gradient into the local estimation

T. T. Lê, A. M. Atto and E. Trouvé are with the Laboratoire d'Informatique, Systèmes, Traitement de l'Information et de la Connaissance (LISTIC), Polytech Annecy-Chambéry, Université de Savoie, B.P. 80439, 74944 Annecy le Vieux Cedex FRANCE (e-mail: thu-trang.le@univ-savoie.fr; abdourrahmane.atto@univ-savoie.fr; emmanuel.trouve@univ-savoie.fr).

J.-M Nicolas is with Laboratoire Traitement et Communication de l'Information (LTCI), Telecom ParisTech, 46 rue Barrault F-75634 Paris Cedex 13, FRANCE (e-mail: jean-marie.nicolas@telecom-paristech.fr).

Digital Object Identifier 10.1109/LGRS.2014.2311663 algorithm [3]. These approaches have better performances compared to boxcar algorithms, but the number of directions is limited. 3) Adaptive neighborhood: These approaches aim at seeking the most suitable neighborhood of each pixel. The adaptive neighborhood can be either a) the maximum homogeneous region (the region including pixels belonging to the same statistical population) selected by testing the evolution of the standard deviation $\sigma$ [4] or the coefficient of variation (CV) [5] or b) the neighborhood consisting of pixels which are similar to the seed pixel (the pixel being processed) [6], [7]. Local estimation within this adaptive neighborhood, hence, yields more accurate results than within fixed neighborhood. However, these methods are time-consuming.

2) Nonlocal approaches, which derive the filtered value by taking into account all the pixels being in the similar context with the considered pixel. Each searching pixel has a weight calculated by a similarity measure between considered patch and searching patch. This idea was first introduced in [8] under the additive white Gaussian noise assumption and has been extended for SAR images in many researches such as: the Probabilistic patch-based (PPB) filter [9], which derives the weights iteratively by using maximum likelihood function. The speckle Bayesian nonlocal (BNL) mean filter [10] resolves the bias assumption problem in the original BNL filter by incorporating Lee improved sigma filter. The SAR Block-matching 3D (SAR-BM3D) filter [11] refines the results obtained from BM3D algorithm by using the local linear minimum mean square error estimator in the wavelet domain and this approach is improved in [12] to speed up block-matching. The spacedomain filter with alterable window (SFAW) [13] is a modified bilateral filter based on local $\mathrm{CV}$ and the joint probability density function model to adapt the window direction by different weights due to different local structures.

With multi-temporal data, both spatial and temporal information can be exploited and this can improve results for filtering SAR data and then, other applications. Several approaches have been developed for multi-temporal filtering, three of them were reviewed and compared in [14]. Most multi-temporal filters have been extended from spatial filters. The filter proposed in [15] is based on a statistical approach: local statistics for each pixel of each image in the time series are estimated by using a spatial window, and then Kuan's filter is applied to obtain the texture compensation of each image. Before deriving filtered images, the summation weights are computed in order to have the optimal variance of the output. This summation weights are then applied in the approach [16]. In [17], the time-space filter uses discrete cosine transform 
(DCT) to transform time axis into frequency axis. Then, a spatial adaptive filter is used to reduce the noise on each frequency channel, except on the zero frequency channel in order to preserve the spatial resolution. Developed from spatial adaptive neighborhood filter, the approach in [18] finds homogeneous regions in a $3 \mathrm{D}$ neighborhood in the volume of piling up registered images, then filtered values are computed on the obtained 3-D neighborhood by using Kuan's filter.

Most multi-temporal filtering approaches have an assumption that spatial pixels remain unchanged over the time and that all of the pixels at the same spatial coordinate in the time series are involved in the filtering process (averaging intensity/amplitude values, for instance). This can be acceptable for some cases such as homogeneous fields, constructions, etc. in short periods, but it is not true for heterogeneous regions and leads to the radiometric degradation in filtered images. In general, this assumption is not appropriate with the fact that the ground features are not stable in all images of the time series, particularly in a long-period time series. To overcome this problem, we propose a novel approach for temporal adaptive filtering of SAR image time series, by integrating change detection technique. The proposed method is based on the detection of changed and unchanged areas derived by applying the $\mathrm{CV}$ test.

This letter is organized into five sections as follows. The next section introduces the strategy proposed for SAR image time series filtering. Section III addresses the properties and implementation of the method. Experimental results are presented in Section IV. Finally, conclusions and perspectives are given in the last section.

\section{Filtering Strategy For SAR Image Time Series}

Let $\mathcal{I}=\left\{\mathcal{I}_{t}\right\}_{1 \leqslant t \leqslant N}$ be an $N$-length coregistered SAR image time series, where $\mathcal{I}_{t}$ denotes the image acquired at time $t$. Let $\mathcal{I}_{t}(i, j)$ be the element located at the $(i, j)$ pixel (line $i$ and column $j$ ) of image $\mathcal{I}_{t}$ and let $\mathcal{I}_{t}^{w}(i, j)$ be an analysis window which determines the boxcar spatial neighborhood of $\mathcal{I}_{t}(i, j)$. Let $\left\{\mathcal{I}_{t}(i, j)\right\}_{1 \leqslant t \leqslant N}$ be a vector including all values of pixels at spatial position $(i, j)$, called pixel pile. Considering $\mathcal{I}$, the proposed approach is made up of a processing chain including three steps.

1) Step 1 - Bi-date analysis: It consists in testing for every two dates $(t, k)_{1 \leqslant t, k \leqslant N}$ the homogeneity of the set $\left\{\mathcal{I}_{t}^{w}(i, j) ; \mathcal{I}_{k}^{w}(i, j)\right\}$.

2) Step 2 - Multidate analysis: Based on the results of the first step, all the homogeneous pixels corresponding to two certain dates $(t, k)_{1 \leqslant t, k \leqslant N}$ are retested to refine the obtained results.

3) Step 3 - Multitemporal filtering: Temporal mean is then applied by taking into account all the homogeneous pixels corresponding to each date issued from the second step to derive filtered images.

\section{A. Bi-date Analysis}

To determine changed and unchanged pixels in each pixel pile, we construct a change detection matrix (CDM) composed of responses of similarity cross tests between each pair of dates. Let $h$ denotes a similarity measure. At pixel position $(i, j)$, considering two pixels of two arbitrary dates $t, k$ in the pixel pile, let

$$
C_{t, k}^{w}(i, j)=h\left(\mathcal{I}_{t}^{w}(i, j), \mathcal{I}_{k}^{w}(i, j)\right)_{1 \leqslant t \leqslant N, 1 \leqslant k \leqslant N}
$$

be the similarity degree between $\mathcal{I}_{t}(i, j)$ and $\mathcal{I}_{k}(i, j)$. To decide whether $C_{t, k}^{w}(i, j)$ represents noise only or contains some change information, we use a threshold $\lambda$. Thus, the change information is:

$M_{t, k}^{w}(i, j)=\left\{\begin{array}{l}0 \text { if } C_{t, k}^{w}(i, j) \leq \lambda \Leftrightarrow \mathcal{I}_{k}^{w}(i, j) \in \Psi\left(\mathcal{I}_{t}^{w}(i, j)\right) \\ 1 \text { if } C_{t, k}^{w}(i, j)>\lambda \Leftrightarrow \mathcal{I}_{k}^{w}(i, j) \notin \Psi\left(\mathcal{I}_{t}^{w}(i, j)\right)\end{array}\right.$

where $\Psi\left(\mathcal{I}_{t}^{w}(i, j)\right)$ is the class of pixel $\mathcal{I}_{t}(i, j)$ including pixels having positive response to the similarity test.

Matrix $M$ is called step 1 Change Detection Matrix (CDM1). This is a symmetric matrix with the size of $N \times N$ containing 0 and 1 values, in which 0 represents "unchanged" and 1 represents "changed".

\section{B. Multidate Analysis}

In order to refine the result of step 1 (changed/unchanged pixel determination in each pixel pile derived from bi-date analysis), we reapply the similarity test given in the previous step but with multidate neighborhood. All homogeneous pixels determined in CDM1 corresponding to each two reference pixels are used to build temporal neighborhoods. In this step, we investigate two cases:

a) For homogeneous regions, the similarity tests are applied with spatio-temporal information of the time series.

The similarity degree between $\Psi\left(\mathcal{I}_{t}^{w}(i, j)\right), \Psi\left(\mathcal{I}_{k}^{w}(i, j)\right)$ is:

$$
\hat{C}_{t, k}^{w}(i, j)=h\left(\Psi\left(\mathcal{I}_{t}^{w}(i, j)\right), \Psi\left(\mathcal{I}_{k}^{w}(i, j)\right)\right)_{1 \leqslant t \leqslant N, 1 \leqslant k \leqslant N}
$$

and it is compared to the threshold $\lambda$ to derive CDM, i.e.,

$$
\hat{M}_{t, k}^{w}(i, j)=\left\{\begin{array}{l}
0 \text { if } \hat{C}_{t, k}^{w}(i, j) \leq \lambda \\
1 \text { if } \hat{C}_{t, k}^{w}(i, j)>\lambda
\end{array}\right.
$$

b) For isolated targets, the changed/unchanged decision is taken by comparing only temporal neighbors of pixels $I_{t}(i, j)$ and $I_{k}(i, j)$ found in the first step, i.e.,

$$
\begin{array}{r}
\hat{C}_{t, k}(i, j)=h\left(\Psi\left(\mathcal{I}_{t}(i, j)\right), \Psi\left(\mathcal{I}_{k}(i, j)\right)\right)_{1 \leqslant t \leqslant N, 1 \leqslant k \leqslant N} \\
\hat{M}_{t, k}(i, j)=\left\{\begin{array}{l}
0 \text { if } \hat{C}_{t, k}(i, j) \leq \lambda \\
1 \text { if } \hat{C}_{t, k}(i, j)>\lambda
\end{array}\right.
\end{array}
$$

Matrix $\hat{M}$ is called step 2 Change Detection Matrix (CDM2) and its form is similar to CDM1.

\section{Multitemporal Filtering}

After step 2, CDM2s provide reliable temporal information on changed and unchanged pixels in each pile. The CDMbased estimator proposed hereafter aggregates the unchanged pixels by using the mean statistics to obtain filtered values, i.e.,

$$
\hat{\mathcal{I}}_{t}(i, j)=\frac{1}{N_{\Psi_{t}(i, j)}} \sum_{k=1}^{N_{\Psi_{t}(i, j)}}\left(1-\hat{M}_{t, k}^{w}(i, j)\right) \mathcal{I}_{k}(i, j)
$$

where $N_{\Psi_{t}(i, j)}$ is the number of elements in class $\Psi\left(\mathcal{I}_{t}(i, j)\right)$. 


\section{ANALYSIS AND IMPLEMENTATION}

\section{A. Analysis of the Estimator}

The estimator given by Eq. (7) has some notable properties.

1) $\hat{\mathcal{I}}_{t}(i, j)$ is the standard unbiased estimator of a nonlocal temporal mean value, applying only on selected pixels (pertaining to the unchanged class).

2) There is no spatial and temporal degradation in the filtered images because the estimator uses only the selected temporal information in the averaging process.

3) At observed pixel pile $(i, j)$, if it is purely unchanged, then the filtered value $\hat{\mathcal{I}}_{t}(i, j)$ is equal to the temporal mean value of the pile. On the opposite, if a pixel in the pile $(i, j)$ is totally different from all other pixels of the pile, then the filtered value $\hat{\mathcal{I}}_{t}(i, j)$ is equal to the original value $\mathcal{I}_{t}(i, j)$. This implies that there is no filtering effect for changed pixel, as expected, for preserving the change information.

4) Considering a time series including $N$ uncorrelated SAR images of $L$-looks, the equivalent number of look (ENL) of the filtered image $\hat{\mathcal{I}}_{t}(i, j)$ is expected to reach $N_{\Psi_{t}(i, j)} \times L$-looks.

The behaviors of the estimator will be illustrated in Sec. IV-B.

\section{B. Implementation}

For implementing the proposed method, the most important thing is the determination of changed and unchanged areas in the time series. This depends on the selection of an appropriate similarity measure and threshold. Several similarity measures have been applied in multi-temporal SAR image change detection, such as Bhattacharyya distance [19], Kullback-Leibler divergence [20], etc. In this letter, we adopt the CV as criterion to test homogeneity of the ground surface reflectivity and an adaptive threshold. The $\mathrm{CV}$ is the ratio of standard deviation to the mean of corrupted image, i.e., $C V=\sigma_{\mathcal{I}} / \mu_{\mathcal{I}}$. The proposed approach aims at detecting the changes in each pixel pile by taking a test using as much as possible temporal samples and a few spatial samples if necessary. The CV can cope with reduced number of samples resulting from small temporal or spatiotemporal neighborhood and an adaptive threshold can deal with the variation of number of samples in each test.

Let us consider an $L$-looks amplitude image $\mathcal{I}$ at pixel position $(i, j)$, i.e.,

$$
\mathcal{I}(i, j)=\rho(i, j) \cdot s(i, j)
$$

where $\rho(i, j)$ is the speckle-free image and $s(i, j)$ is the speckle noise. Standard deviation of speckle noise in purely homogeneous area is $\sigma_{s}=0.5227 / \sqrt{L}$. If the ground surface is purely homogeneous, then $C V=\sigma_{s}$; on the contrary, if it varies, $C V>\sigma_{s}$. The $\mathrm{CV}$ test is represented as

$$
C V(i, j) \leq \sigma_{s}+\delta(i, j)
$$

where $\delta(i, j)$ is a small value denoting a tolerance of the threshold, derived from [21]. Thus, the adaptive threshold is

$$
\lambda(i, j)=\eta\left(\sigma_{s}+\sigma_{s} \sqrt{\frac{1+2 \sigma_{s}^{2}}{2 n(i, j)}}\right)
$$

in which $n(i, j)$ is the number of elements involved in the $C V(i, j)$ test, $\eta$ is a system parameter determining the degree of smoothing. In general, the values of $\eta$ are around 1.0 [21].

\section{EXPERIMENTAL RESULTS}

\section{A. Test-site Data}

The proposed approach is illustrated on a time series of 25 single look complex ascending TerraSAR-X images acquired from November 6, 2009 to September 25, 2011 over Chamonix-MontBlanc with 2-m resolution. We study two characteristic test-sites: a parking area (Grands Montets) and a moving glacier (Argentière) which surface moves about 20 $\mathrm{cm} /$ day, i.e., more than one pixel between two consecutive dates in the time series. The two test-sites include different kinds of change: parking occupation, glacier surface evolution, etc. (see Fig. 1).

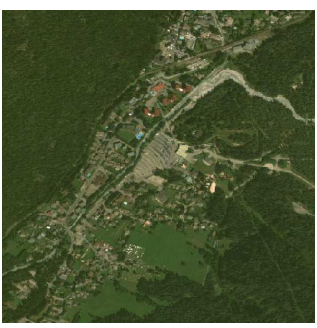

(a)

(b)

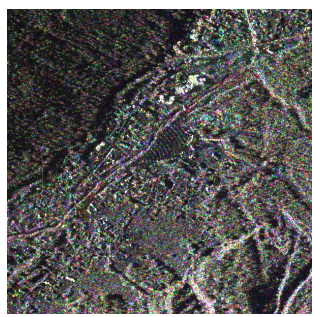

Fig. 1. Chamonix-MontBlanc test-site. (a): optical image, (b): TerraSAR-X color composition image (R: 06/07/2011; G: 06/18/2011; B: 06/29/2011); left: Grands Montets parking, right: Argentière glacier.

\section{B. Temporal Adaptive Filtering Results}

In this letter, we apply the minimum analysis window (including central pixel and its four nearest neighbors) when using spatial information. For homogeneous regions such as grass, Fig. 2 (a) and (b) shows that the proposed filtering operator can detect unchanged pixels in each pixel pile effectively. CDM2 detects more unchanged pixels than CDM1 does. The profiles of filtered time series using CDM1 and CDM2 in Fig. 2 (c) are quite correlated to the one of original time series but the dynamic range of amplitude pixel values in filtered time series are reduced due to the averaging process in step 3 of the proposed method, and it also reduces speckle noise significantly.

In the case of isolated target such as a car in the parking, there is only one date (one image) in which an object appears at a certain location. The pixel at the object location of that date is completely different from other pixels in the pixel pile. After filtering, the pixel value of the object is preserved as illustrated in Fig. 3.

Looking into Fig. 4, the reduction of speckle in filtered image helps us to see clearly details of the parking with small 
(a)

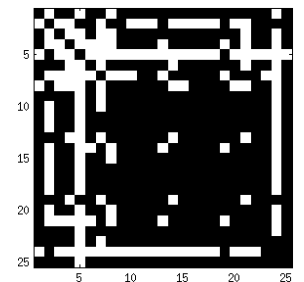

(c)

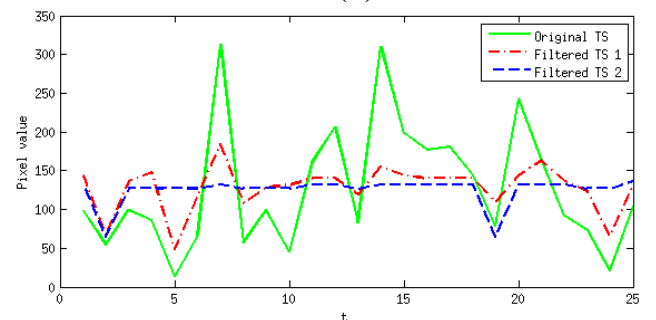

Fig. 2. Change detection matrices CDM1 (a), CDM2 (b) and temporal profiles (c) for a pixel in a homogeneous region (grass) of original time series (TS) in solid line, filtered TS using CDM1 (filtered TS 1) in dash-dot line and using CDM2 (filtered TS 2) in dashed line.

(a)

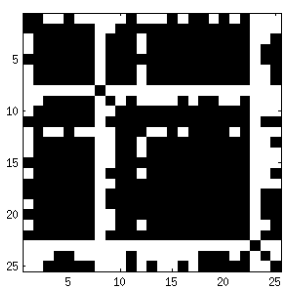

(c)

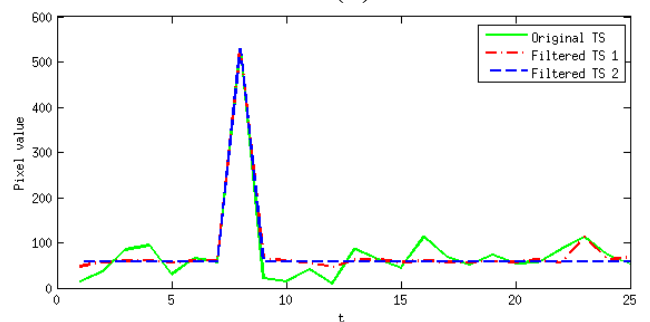

Fig. 3. Change detection matrices CDM1 (a), CDM2 (b) and temporal profiles (c) for a pixel pile with isolated target (a car in the parking).

cars, which cannot be distinguished in the original image. Fig. 5 reveals another interesting point of this filter: it removes well speckle in unchanged areas but it almost avoids filtering moving areas. The filtering reduces the speckle in the stable rock area, whereas in the glacier area, original and filtered images look quite similar. In this area, glacier pixel values in the time series change from date to date, and each point on this surface moves more than one pixel between different acquisitions. Therefore, the filter which is based on averaging unchanged pixels detects very few unchanged samples for the averaging process.

\section{Performance Assessment}

The performance of a multitemporal filter is assessed through noise reduction, spatial information (texture, edge, size, etc.) and temporal information (change) preservation.

Histograms of CDMs at different characteristic regions in Fig. 6 illustrate that the proposed filter preserves changes well. At a stable grass area, almost all pixels in piles of this area are unchanged; on the average, the CDM2 histogram mode

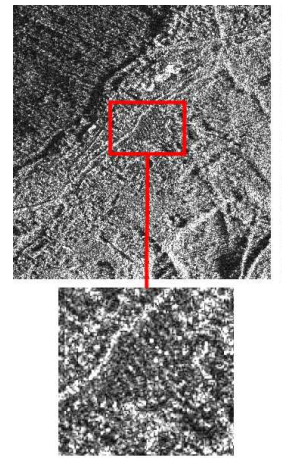

(a)

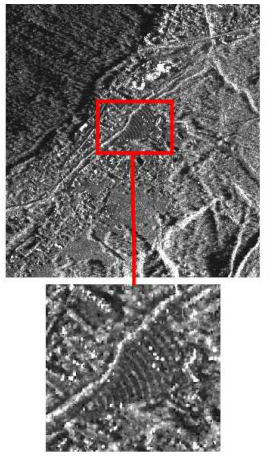

(b)

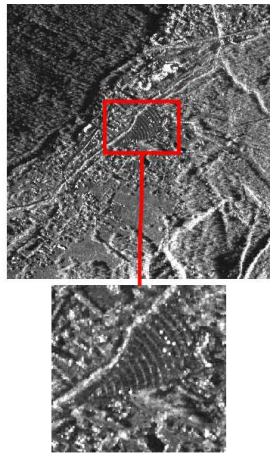

(c)
Fig. 4. Filtering results at parking test-site. Original image (08/27/2009) (a), filtered image using CDM1 (b) and CDM2 (c).

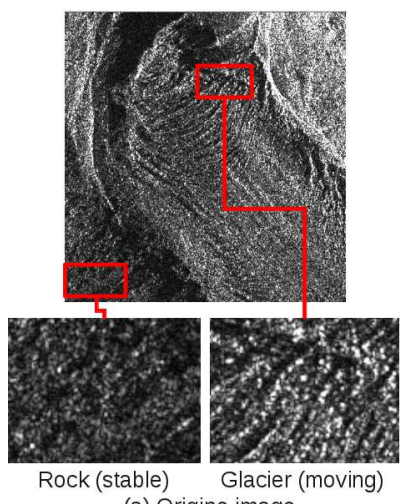

(a) Origine image

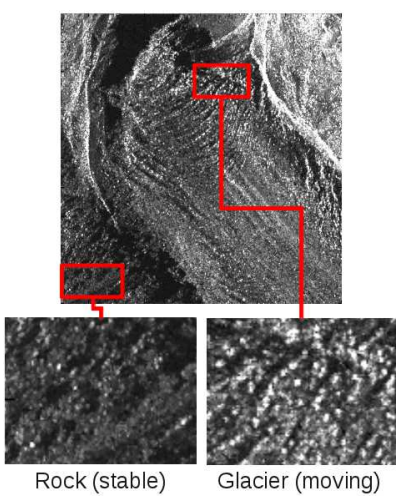

(b) Filtered image
Fig. 5. Filtering results at glacier test-site, image acquired on 09/03/2011.

highlights that approximately 20 filtered values are temporal mean of a whole pixel pile. With the piles of forest area where pixels change seasonally leading to some radiometric changes (unfiltered) and some unchanged sequences in each pile, the maximum number of unchanged pixels of piles is about 17 . At a moving area, about 7 original pixels are unfiltered in general, and the mean values of small numbers of samples are calculated to derive the rest of the filtered values.

To assess speckle reduction and spatial information preservation, the proposed filter is compared with another multitemporal filtering approach proposed by Quegan et al. [16] by employing equivalent number of look (ENL) criterion and blind/referenceless image spatial quality evaluator (BRISQUE)

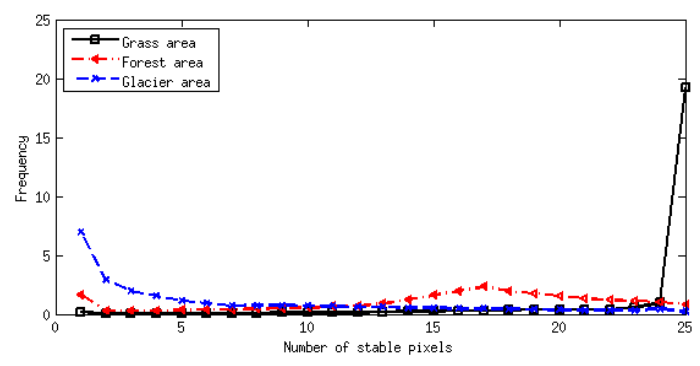

Fig. 6. Histograms of CDM2s at different characteristic regions. 
model [22]. BRISQUE model quantifies possible losses of "naturalness" in the image due to the presence of distortions, and it has a value between 0 and 100 ( 0 represents the best quality). Table I presents ENL and BRISQUE indexes of filtered time series obtained by Quegan's filter (QF) and CDM-based filter (CDMF). CDMF time series' ENLs increase significantly in comparison with ENLs of the original time series and are higher than those of the QF time series. BRISQUE indexes of the CDMF time series are at good level and better than those of the QF time series results.

TABLE I

IMAGE QUALITY INDEXES.

\begin{tabular}{||c||c|c|c||c|c||}
\hline \hline \multirow{2}{*}{\multicolumn{1}{|c||}{ No }} & \multicolumn{3}{c|}{ ENL } & \multicolumn{2}{c||}{ BRISQUE } \\
\cline { 2 - 6 } & Origin & QF & CDMF & QF & CDMF \\
\hline \hline 1 & 0.9341 & 2.7178 & 12.4390 & 34.3285 & 29.7607 \\
\hline 2 & 0.8936 & 2.8005 & 12.2835 & 33.5157 & 29.6305 \\
\hline 3 & 0.8765 & 2.7880 & 13.5580 & 36.1276 & 28.5864 \\
\hline 4 & 0.8824 & 2.6486 & 13.6410 & 34.2162 & 28.9878 \\
\hline 5 & 0.8524 & 2.3941 & 12.4541 & 34.3133 & 30.6759 \\
\hline 6 & 0.9578 & 3.0453 & 12.8848 & 34.5963 & 28.3462 \\
\hline 7 & 0.9137 & 2.6718 & 13.2406 & 33.6967 & 29.3074 \\
\hline 8 & 0.9369 & 2.7075 & 12.7328 & 33.8096 & 29.7147 \\
\hline 9 & 0.8484 & 2.3656 & 13.4446 & 34.1750 & 28.7904 \\
\hline 10 & 0.9323 & 2.8827 & 13.3715 & 34.9807 & 27.9359 \\
\hline 11 & 0.9432 & 3.0015 & 10.7973 & 35.2731 & 28.7787 \\
\hline 12 & 0.8725 & 2.5637 & 13.8458 & 33.3171 & 27.1596 \\
\hline 13 & 0.9202 & 2.7853 & 13.4212 & 34.2332 & 26.7577 \\
\hline 14 & 0.9303 & 2.7100 & 12.3830 & 35.2837 & 27.9568 \\
\hline 15 & 0.9818 & 3.1286 & 13.2644 & 35.7331 & 27.7234 \\
\hline 16 & 1.0004 & 3.0793 & 13.1577 & 34.4976 & 26.7082 \\
\hline 17 & 0.9758 & 3.0305 & 11.4058 & 33.7041 & 28.1111 \\
\hline 18 & 0.9225 & 2.9147 & 10.8909 & 32.8023 & 30.2470 \\
\hline 19 & 0.9617 & 2.9940 & 13.6586 & 35.1139 & 28.0325 \\
\hline 20 & 0.9730 & 3.0845 & 11.8362 & 35.6142 & 27.2652 \\
\hline 21 & 0.9481 & 2.7420 & 11.3806 & 34.2447 & 28.2388 \\
\hline 22 & 0.9187 & 2.8617 & 13.5367 & 35.1608 & 27.4113 \\
\hline 23 & 0.9425 & 2.8704 & 13.4048 & 34.3715 & 27.7962 \\
\hline 24 & 1.0228 & 3.2788 & 12.9309 & 35.5937 & 28.3087 \\
\hline 25 & 0.8825 & 2.6883 & 13.2800 & 33.6532 & 27.0954 \\
\hline \hline Mean & $\mathbf{0 . 9 2 9 0}$ & $\mathbf{2 . 8 3 0 2}$ & 12.7698 & $\mathbf{3 4 . 4 9 4 2}$ & $\mathbf{2 8 . 3 7 3 1}$ \\
\hline \hline
\end{tabular}

\section{CONCLUSIONS}

In this letter, a new method for temporal adaptive filtering of SAR image time series is presented. The proposed estimator is based on the determination of changed and unchanged pixels. Indeed, for a heterogeneous scene composed of stable and dynamic areas, multitemporal filtering is unachievable if the information of change detection is not taken into account. The experimental results have shown that the proposed approach reduces speckle significantly while preserving detail features: edges and changes. The filter has very little impact on moving areas. This advantage allows images including stable and moving areas to be filtered without losing characteristics of moving areas that are very important in monitoring their evolution (glacier for instance) and simultaneously reduces speckle at other areas of images.

Due to the results of this approach, further works may concern: developing an automatic test for purely temporal or spatiotemporal neighborhood and analyzing multitemporal change in time series using CDM. The information of CDM indeed opens abilities to make change detection multimap and to do not only spatial but also temporal segmentation.

\section{ACKNOWLEDGMENT}

The authors would like to thank the German Aerospace Center (DLR) for providing the TerraSAR-X time series used in the experiments under the project MTH0232.

\section{REFERENCES}

[1] J. S. Lee, "Digital image enhancement and filtering by use of local statistics," IEEE Trans. Pattern Anal. Mechine Intell., vol. 7(2), pp. 165168,1980

[2] D. T. Kuan, A. A. Sawchuk, T. C. Strand, and P. Chavel, "Adaptive restoration of images with speckle," IEEE Trans. Acoust., Speech and Sign. Process., vol. 35, pp. 373-383, 1987.

[3] J.-S. Lee, "Refined filtering of image noise using local statistic," Compt. Graph. Image Process, vol. 15, pp. 380 - 389, 1981.

[4] Y. Wu and H. Maitre, "Smoothing speckled synthetic aperture radar images by using maximum homogeneous region filters," Opt. Eng, vol. 31, no. 8, pp. 1782-1792, 1992.

[5] J. M. Nicolas, F. Tupin, and H. Maitre, "Smoothing speckled sar images by using maximum homogeneous region filters: An improved approach," in Proc. IGARSS Sydney, Australia, vol. III, p. 1503-1505, 2001.

[6] R. M. Rangayyan, M. Ciuc, and F. Faghih, "Adaptive-neighborhood filtering of images corrupted by signal-dependent noise," Appl. Opt., vol. 37, no. 20, pp. 4477-4487, 1998.

[7] G. Vasile, E. Trouve, J.-S. Lee, and V. Buzuloiu, "Intensity-driven adaptive-neighborhood technique for polarimetric and interferometric SAR parameters estimation," IEEE Trans. Geosci. Remote Sens., vol. 44 , no. 6, pp. 1609-1621, 2006.

[8] A. Buades, B. Coll, and L. M. Morel, "A review of image denoising algorithms, with a new one," SIAM Interdisc. J.: Multiscale Model. Simul., vol. 4, no. 2, pp. 490-530, 2005.

[9] C.-A. Deledalle, L. Denis, and F. Tupin, "Iterative weighted maximum likelihood denoising with probabilistic patch-based weights," IEEE Trans. Geosci. Remote Sens., vol. 18, no. 12, pp. 2661-2672, 2009.

[10] H. Zhong, Y. Li, and L. Jiao, "SAR image despeckling using bayesian nonlocal means filter with sigma preselection," IEEE Geosci. Remote Sens. Lett., vol. 8, no. 4, pp. 809-813, 2011.

[11] S. Parrilli, M. Poderico, C. V. Angelino, and L. Verdoliva, "A nonlocal SAR image denoising algorithm based on LLMMSE wavelet shrinkage," IEEE Trans. Geosci. Remote Sens., vol. 50, no. 2, pp. 606-616, 2012.

[12] D. Cozzolino, S. Parrilli, G. Scarpa, G. Poggi, and L. Verdoliva, "Fast adaptive nonlocal SAR despeckling," IEEE Geosci. Remote Sens. Lett., 2013.

[13] G.-T. Li, C.-L. Wang, P.-P. Huang, and W.-D. Wu, "SAR image despeckling using a space-domain filter with alterable window," IEEE Geosci. Remote Sens. Lett., vol. 10, no. 2, pp. 263-267, 2013.

[14] E. Trouve, Y. Chambenoit, N. Classeau, and P. Bolon, "Statistical and operational performance assessment of multitemporal SAR image filtering." IEEE Trans. Geosci. Remote Sens., vol. 41, no. 11, pp. 25192530, 2003.

[15] J. Bruniquel and A. Lopes, "Multi-variate optimal speckle reduction in SAR imagery," Int. J. Remote Sens., vol. 18, no. 3, pp. 603-627, 1997.

[16] S. Quegan, T. L. Toan, J. J. Y. Jiong, F. Ribbes, and N. Floury, "Multitemporal ERS SAR analysis applied to forest mapping," IEEE Trans. Geosci. Remote Sens., vol. 38 , no. 2, pp. 741-753, 2000.

[17] D. Coltuc, E. Trouve, F. Bujor, N. Classeau, and J. P. Rudant, "Timespace filtering of multitemporal SAR images," in Proc. IGARSS Honolulu, HI, vol. 7, pp. 2909-2911, 2000.

[18] M. Ciuc, P. Bolon, E. Trouve, V. Buzuloiu, and J.-P. Rudant, "Adaptiveneighborhood speckle removal in multitemporal SAR images," Appl. Opt., vol. 40, no. 32, pp. 5954-5966, 2001.

[19] J. Morio, P. Refregier, F. Goudail, P. C. Dubois-Fernandez, and $\mathrm{X}$. Dupuis, "Information theory-based approach for contrast analysis in polarimetric and/or interferometric SAR images," IEEE Trans. Geosci. Remote Sens., vol. 46, no. 8, pp. 2196-2185, 2008.

[20] J. Inglada and G. Mercier, "A new statistical similarity measure for change detection in multitemporal SAR images and its extension to multiscale change analysis," IEEE Trans. Geosci. Remote Sens., vol. 45, no. 5, pp. 1432-1445, 2007.

[21] J.-M. Park, W. J. Song, and W. A. Pearlman, "Speckle filtering of SAR images based on adaptive windowing," IEE Proc Vis. Image Sign. Process, vol. 146, no. 4, pp. 191-197, 1999.

[22] A. Mittal, A. K. Moorthy, and A. C. Bovik, "No-reference image quality assessment in the spatial domain," IEEE Trans. Image Process, vol. 21 , no. 12 , pp. $4695-4708,2012$. 